\title{
Room temperature negative differential resistance of a monolayer molecular rotor device
}

\author{
Mei Xue, ${ }^{1, a)}$ Sanaz Kabehie, ${ }^{2}$ Adam Z. Stieg, ${ }^{3}$ Ekaterina Tkatchouk, ${ }^{4}$ Diego Benitez, ${ }^{4}$ \\ Rachel M. Stephenson, ${ }^{2}$ William A. Goddard, ${ }^{4}$ Jeffrey I. Zink, ${ }^{2,3}$ and Kang L. Wang ${ }^{1,3}$ \\ ${ }^{1}$ Department of Electrical Engineering, Device Research Laboratory, University of California, Los Angeles, \\ California 90095, USA \\ ${ }^{2}$ Department of Chemistry and Biochemistry, University of California, Los Angeles, California 90095, USA \\ ${ }^{3}$ California NanoSystems Institute, University of California, Los Angeles, California 90095, USA \\ ${ }^{4}$ Materials and Process Simulation Center, California Institute of Technology, Pasadena, \\ California 91125, USA
}

(Received 17 June 2009; accepted 12 August 2009; published online 2 September 2009)

\begin{abstract}
An electrically driven molecular rotor device comprised of a monolayer of redox-active ligated copper compounds sandwiched between a gold electrode and a highly doped $P^{+} \mathrm{Si}$ substrate was fabricated. Current-voltage spectroscopy revealed a temperature-dependent negative differential resistance (NDR) associated with the device. Time-dependent density functional theory suggests the source of the observed NDR to be redox-induced ligand rotation around the copper metal center, an explanation consistent with the proposed energy diagram of the device. An observed temperature dependence of the NDR behavior further supports this hypothesis. (C) 2009 American Institute of Physics. [DOI: 10.1063/1.3222861]
\end{abstract}

Negative differential resistance (NDR) is an essential property that allows fast switching in certain types of electronic devices such as Esaki diode and IMPATT diode. ${ }^{1,2}$ Beyond the traditional electronic devices, a number of nanoscale molecular devices have been studied and reported to exhibit NDR characteristics. ${ }^{3-7}$ Although various mechanisms have been proposed, including charging and discharging processes of electrons, chemical reaction, redox reaction, and the association-dissociation processes of molecules, their specific underlying physics remain unclear. These functional molecular units acting as state variables provide an attractive alternative to overcome the limits of conventional metaloxide-semiconductor field-effect transistor technology due to their potential scalability, low cost, low variability, highly integrateable characteristics, and the capability to exploit self-assembly processes. ${ }^{6-10}$ Thus the elucidation of switching mechanisms and the development of different operational approaches have drawn a lot of attention. In this letter, we demonstrate an electrically driven sandwich-type monolayer molecular rotor switch with NDR. The observed NDR behavior is attributed to rotational motion on solid support. Both calculations of time-dependent density functional theory and an observed temperature dependence of the NDR behavior support this hypothesis.

Synthesized using a self-assembly approach, the molecular switch device shown in Fig. 1 is comprised of a heteroleptic copper compound covalently bonded to a highly doped silicon substrate. Each complex contains three subunits: a bifunctional stator [a bidentate ligand bonded to both a solid support $\left(P^{+} \mathrm{Si}\right)$ and a $\mathrm{Cu}$ axle], the metal axle $(\mathrm{Cu})$, and a diimine rigid rotator (2,9-dimethyl-1,10-phenanthroline). Preparation of the heteroleptic copper system was carried out through covalent grafting of a stator monolayer onto the hydroxylated surface of a $P^{+} \mathrm{Si}$ substrate using silanol bonds. ${ }^{11}$

\footnotetext{
a) Author to whom correspondence should be addressed. Tel.: +1-310-2060207. FAX: +1-310-206-8495. Electronic mail: mxue@ee.ucla.edu.
}

The stators were then used to chelate a copper metal axle that subsequently bonds to the rotator subunit. The device was completed by deposition of a Ti/gold film through a shadow mask on top of the molecular layer to form the top electrode. The number of molecules per unit area and per unit devices tested is approximately $1.1 \times 10^{14} \mathrm{~cm}^{-2}$ and $8 \times 10^{10}$, respectively. This copper compound exhibits two discrete, redox-dependent conformational states, $\mathrm{Cu}(\mathrm{I})$ and $\mathrm{Cu}(\mathrm{II})$. $\mathrm{The} \mathrm{Cu}(\mathrm{I})$ form has tetrahedral geometry while the $\mathrm{Cu}(\mathrm{II})$ form is square planar. ${ }^{12}$ The compounds undergo a one electron redox-induced rotational conformational change depending on the oxidation state of the copper metal. Interconversion between these two states provides the basis for a controlled, bistable nanoswitch.

$I-V$ characteristics of the monolayer device are shown in Fig. 2. In this case, the silicon substrate represents the system ground and the dc bias applies from the top gold electrode. The bias sweep direction followed the arrows numerically as shown in Fig. 2. Unlike previously reported devices, the NDR observed here disappeared after reversal of the sweep-

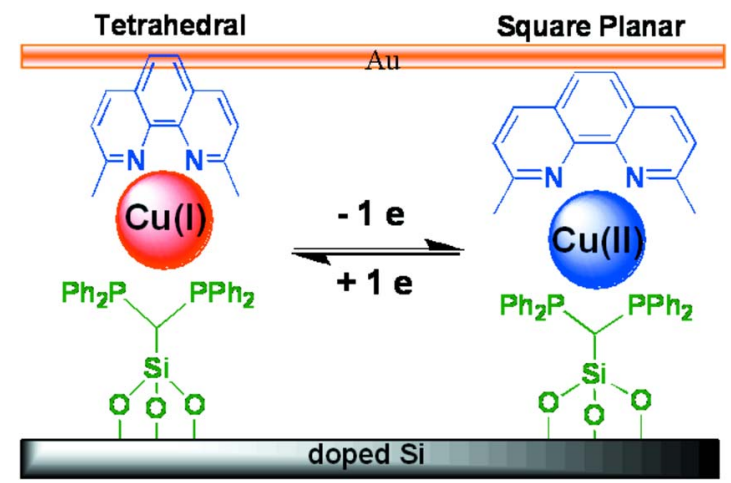

FIG. 1. (Color online) The schematic structure of the molecular rotor device composed of a bisP stator, a copper metal axle, and a diimine rotator. The diimine ligand on top, 2,9-dimethyl-1,10-phenanthroline, rotates upon reduction and oxidation in the direction as illustrated with arrows. 


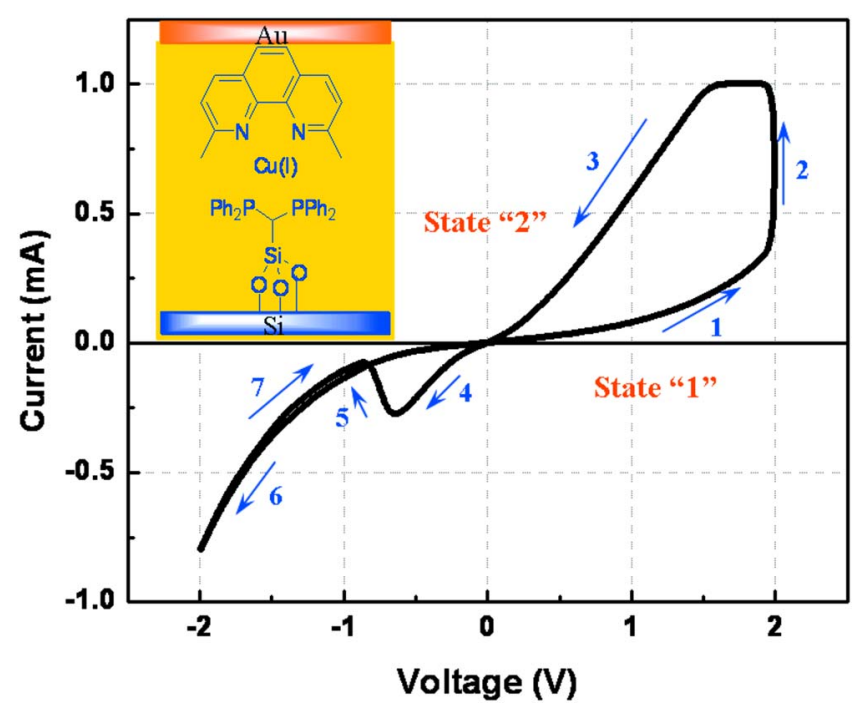

FIG. 2. (Color online) $I-V$ characteristics of the monolayer device with the bisP stator. State "1" represents the low conductivity state, while state " 2 " is the high conductivity state. The turning voltage between state " 1 " and state " 2 " is determined by the redox energy of the copper system. The difference in value of the turning voltage between positive and negative ranges is due to the different contact energy barriers of the two electrodes. The arrows show the sequence of the voltage scan. Arrows "4" and "5" correspond to the band diagrams in Figs. 4(a) and 4(b), respectively (to be discussed later).

ing direction in the negative voltage regime (arrow 7). At room temperature, the peak current density is approximately $0.1 \mathrm{~A} / \mathrm{cm}^{2}$ with a corresponding peak-to-valley ratio near 5 . For a given device, the $I-V$ characteristics are stable and reproducible with consecutive positive and negative bias sweeps. The magnitude of observed current fluctuations is smaller than $1 \%$ and errors are within $0.5 \%$. Small fluctuations are observed among devices owing to the nonuniformity of the active molecular layer.

To further explore the correlation of the observed NDR effect with redox-induced conformational switching, devices whose molecular layer consisted of solely grafted stators or $\mathrm{Cu}$ ligated stators were prepared. Due to their lack of a ligated rigid rotator, these devices lack the capability for rotational motion upon oxidation or reduction of the $\mathrm{Cu}$ metal axle. The lack of observed NDR effects in these cases implies that the source of the observed NDR in the full device is due to the conformational change in the active molecular layer. As seen in Fig. 2, state "1" and state "2" represent the low and high conductivity states, respectively. The turning voltage between state " 1 " and state " 2 " is determined by the redox energy of the copper system. Variations in the turning voltages at positive and negative bias are attributed to differences in the contact energy barriers of the two electrodes.

To gain a thorough understanding of electron transport during this rotation behavior, temperature dependent $I-V$ measurements of the device were performed from $77 \mathrm{~K}$ to room temperature. The NDR effect disappeared at about $<244 \mathrm{~K}$. The peak minus background currents at multiple temperatures were extracted and plotted as shown in Fig. 3. Peak currents measured at $T>244 \mathrm{~K}$ followed an exponential relationship with respect to the reciprocal of the temperature. Based on the well-known Arrhenius equation, we have

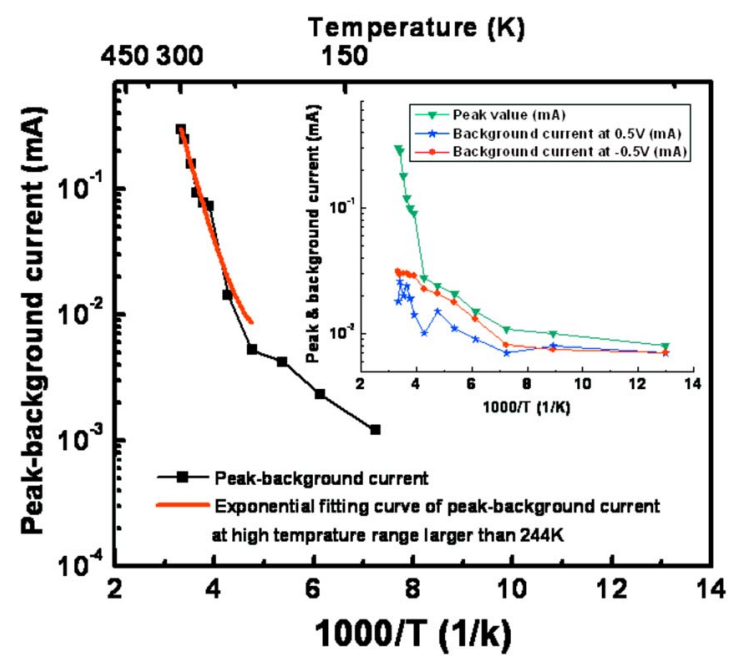

FIG. 3. (Color online) Arrhenius plot of the peak minus background current. The activation energy of rotation was extracted to be $0.3 \mathrm{eV}$ by exponentially fitting the curve of the measurement data higher than $244 \mathrm{~K}$. The inset shows the peak current as well as the background current at $0.5 \mathrm{~V}$ and -0.5 $\mathrm{V}$, respectively. The weak temperature dependence of the background current implies tunneling transport of electrons.

$$
E_{a}=-R\left[\frac{\partial \ln k}{\partial(1 / T)}\right]_{P}
$$

where $E_{\mathrm{a}}$ is the activation energy, $k$ is the rate constant of chemical reactions, $T$ is the temperature, and $R$ is the gas constant. Curve fitting of the data acquired for $T>244 \mathrm{~K}$ yielded a rotational activation energy of approximately 0.3 $\mathrm{eV}$, and this value is consistent with the theoretical quenching energy of the rotation in solution. ${ }^{13,14}$ This solid agreement between the extracted activation energy and the theoretical quenching energy supports the proposal that the observed NDR effect in this device is due to rotational motion within the molecular layer. The peak and background currents at 0.5 and $-0.5 \mathrm{~V}$, respectively, were plotted versus $1000 / T$ and displayed different temperature dependences, as shown in the inset of Fig. 3. The weak temperature dependence of both background currents is indicative of electron tunneling between the two device electrodes.

The proposed mechanism for the observed device $I-V$ characteristics shown in Fig. 2 is based on an electron tunneling induced molecular rotation behavior that serves to modify the band diagram of the active molecular layer. Density Functional Theory (DFT) calculations have been employed in an attempt to reconcile the sequence of carrier transport processes and the role of energy states, ${ }^{15}$ which helped formulate the proposed band diagram presented in Fig. 4. Application of a bias potential to the top gold electrode shifts the energy positions of molecular states relative to both gold electrode and $P^{+} \mathrm{Si}$ band edges. As the rotary motion around the molecular axle is controlled by electron transfer, a portion of the applied potential is used to initiate the redox process within the molecular layer. First, theoretical calculations ${ }^{16}$ of the highest occupied molecular orbital (HOMO)-lowest unoccupied molecular orbital (LUMO) band gap for the $\mathrm{Cu}(\mathrm{I})$ and $\mathrm{Cu}(\mathrm{II})$ complexes by timedependent DFT (TD-DFT) yielded values of approximately 2.7 and $3.4 \mathrm{eV}$, respectively. Similar methods have been used in the study of organocopper complexes. ${ }^{17-20}$ Additional calculations of the molecular redox energy produced a value of 

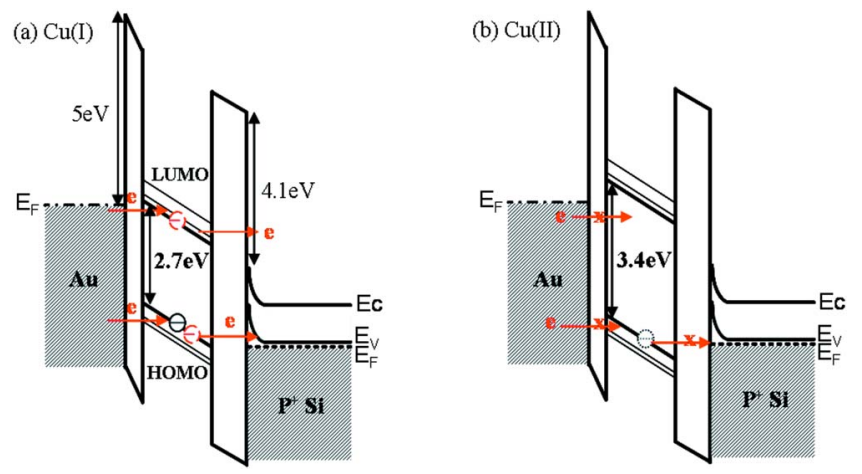

FIG. 4. (Color online) Band diagram of the molecular rotor device under negative bias at (a) high conductivity state of $\mathrm{Cu}(\mathrm{I})$ system and (b) low conductivity state of $\mathrm{Cu}$ (II) system. As oxidation happens, one electron in the valence band tunnels out of the molecular layer and the energy states move down. The band gap increases so that the conduction path for electrons is closed, resulting in NDR.

$1.05 \mathrm{eV}$, which is close to $0.7 \mathrm{eV}$ of the difference of band gaps between $\mathrm{Cu}(\mathrm{I})$ and $\mathrm{Cu}(\mathrm{II})$. The $0.35 \mathrm{eV}$ difference between these two values may be attributed to the rotational energy contribution. Finally, the electron affinity of the $\mathrm{Cu}(\mathrm{I})$ complex is estimated to be $3.7 \mathrm{eV}$, which anchors the LUMO energy state with respect to the gold Fermi level.

The process begins with the low conductivity state of the $\mathrm{Cu}$ (II) complex under positive bias (arrow 1 in Fig. 2). The device remains in the low conductivity state until one electron from the $P^{+} \mathrm{Si}$ substrate tunnels through the energy barrier and charges into the HOMO state of the active molecular layer. The resulting reduction of $\mathrm{Cu}$ (II) to $\mathrm{Cu}$ (I) shifts the relative position of the HOMO energy states upward and serves to shrink the band gap, resulting in a high current (arrow 2 in Fig. 2). Upon application of negative bias to the gold electrode, the conducting channel of the $\mathrm{Cu}(\mathrm{I})$ complex remains open and results in current flow through both HOMO and LUMO states shown as Fig. 4(a) (arrow 4 in Fig. 2). Subsequent oxidation of $\mathrm{Cu}$ (I) to $\mathrm{Cu}$ (II) occurs when one electron tunnels out of the HOMO state and discharges the active molecular layer. This process alters the band structure and increases the band gap due to a reduction in Coulomb energy by the removal of one electron shown as Fig. 4(b) (arrow 5 in Fig. 2). Current flow then stops, resulting in NDR. The return trace does not show an NDR behavior as the conduction path is closed. Continuing toward more negative bias produced an exponential increase in measured current with respect to the applied electric field, which is attributed to direct tunneling between the two electrodes. Since the model used in the calculations was based on the gas phase, discrepancies between the calculated and the experimental values could be the result of deviations from the theoretical model due to adsorption of the molecules onto a solid support. There are other possible transport mechanisms ${ }^{21}$ such as Redox-based filament, hopping transport, which one might consider. For both of these two transport mechanisms, the current would show different temperature dependences. ${ }^{22,23}$ However for our device, the weak temperature dependence of the background current implies the tunneling transport. Furthermore, the thickness of the molecular layer in our device is only $<5 \mathrm{~nm}$, and thus the filament formation is not likely. ${ }^{24}$ In addition, the control samples with $\mathrm{Zn}$ as a metal axel, which cannot be rotated with the redox process, did not show any NDR effects. The above arguments further disqualify the filament transport as the conduction mechanism in our molecular rotor devices. Thus we may conclude that our proposed Redox-assisted tunneling transport is the most likely model to explain the observed phenomena. Clearly further experiments are needed in order to gain deeper understanding of the underlying mechanism for this molecular device.

In summary, an electrically driven molecular rotor device has been designed and fabricated. The NDR behavior was observed and attributed to the rotational motion about the copper metal axle. From temperature dependent measurements, the activation energy of the rotation was estimated to be $0.3 \mathrm{eV}$ from the Arrhenius plot. The proposed band diagram was used to explain the electron transport behavior during the device operation.

The authors would like to thank the FCRP-FENA center for support.

${ }^{1}$ L. Esaki, Phys. Rev. 109, 603 (1958).

${ }^{2}$ L. L. Chang, L. Esaki, and R. Tsu, Appl. Phys. Lett. 24, 593 (1974).

${ }^{3}$ J. Chen, M. A. Reed, A. M. Rawlett, and J. M. Tour, Science 286, 1550 (1999).

${ }^{4}$ Y. Xue, S. Datta, S. Hong, R. Reifenberger, J. I. Henderson, and C. P. Kubiak, Phys. Rev. B 59, R7852 (1999).

${ }^{5}$ C. B. Gorman, R. L. Carroll, and R. R. Fuierer, Langmuir 17, 6923 (2001).

${ }^{6}$ A. Aviram and M. A. Ratner, Chem. Phys. Lett. 29, 277 (1974).

${ }^{7}$ N. Tao, Nat. Nanotechnol. 1, 173 (2006).

${ }^{8}$ S. M. Lindsay and M. A. Ratner, Adv. Mater. 19, 23 (2007).

${ }^{9}$ C. Joachim, J. K. Gimzewski, and A. Aviram, Nature (London) 408, 541 (2000)

${ }^{10}$ J. K. Gimzewski, C. Joachim, R. R. Schlittler, V. Langlais, H. Tang, and I. Johannsen, Science 281, 531 (1998).

${ }^{11}$ F. Piestert, R. Fetouaki, M. Bogza, T. Oeser, and J. Blumel, Chem. Commun. (Cambridge) 2005, 1481.

${ }^{12}$ F. A. Cotton, W. Geoffrey, C. A. Murillo, and M. Bochmann, Advanced Inorganic Chemistry, 6th ed. (Wiley, New York, 1999).

${ }^{13}$ D. V. Scaltrito, D. W. Thompson, J. A. Ocallaghan, and G. J. Meyer, Coord. Chem. Rev. 208, 243 (2000).

${ }^{14}$ L. X. Chen, G. B. Shaw, I. Novozhilova, T. Liu, G. Jennings, K. Attenkofer, G. J. Meyer, and P. Coppens, J. Am. Chem. Soc. 125, 7022 (2003).

${ }^{15}$ J. M. Seminario, A. G. Zacarias, and J. M. Tour, J. Am. Chem. Soc. 122, 3015 (2000).

${ }^{16}$ All calculations were performed on model system $\left[\mathrm{Cu}(2,9 \text {-dimethyl-1,10-phenanthroline })_{2}\right]^{x+}$ in the gas phase with Gaussian 03 using the density functional B3LYP with the 6-31G(d) basis set. (Frisch, M. J. Gaussian 03, revision C.02; Gaussian, Inc.: Wallingford, CT, 2004.) The LANL2DZ basis set was used for $\mathrm{Cu}$ and the $6-31 \mathrm{G}(\mathrm{d}, \mathrm{p})$ basis set for $\mathrm{C}, \mathrm{N}$, and $\mathrm{H}$.

${ }^{17}$ J. Cody, J. Dennisson, J. Gilmore, D. G. VanDerveer, M. M. Henary, A. Gabrielli, C. D. Sherrill, Y. Y. Zhang, C. P. Pan, C. Burda, and C. J. Fahrni, Inorg. Chem. 42, 4918 (2003).

${ }^{18}$ X. J. Wang, C. Lv, M. Koyama, M. Kubo, and A. Miyamoto, J. Organomet. Chem. 690, 187 (2005).

${ }^{19}$ L. Yang, J. K. Feng, A. M. Ren, M. Zhang, Y. G. Ma, and X. D. Liu, Eur. J. Inorg. Chem. 2005, 1867 (2005).

${ }^{20}$ M. Z. Zgierski, J. Chem. Phys. 118, 4045 (2003).

${ }^{21}$ J. C. Scott and L. D. Bozano, Adv. Mater. 19, 1452 (2007)

${ }^{22}$ G. W. Dietz, W. Antpohler, M. Klee, and R. Waser, J. Appl. Phys. 78, 6113 (1995).

${ }^{23}$ N. F. Mott and E. A. Davis, Electronic Processes in Non Crystalline Materials (Clarendon, London, 1979), p. 404.

${ }^{24}$ R. Waser and M. Aono, Nature Mater. 6, 833 (2007). 